2011-09-20

\title{
Creating a Uniform Magnetic Field for the Equi-Biaxial Physical Testing of Magnetorheological Elastomers; Electromagnet Design, Development and Testing.
}

\author{
Dave Gorman \\ Technological University Dublin, david.gorman@tudublin.ie \\ Stephen Jerrams \\ Technological University Dublin, stephen.jerrams@tudublin.ie \\ Ray Ekins \\ Technological University Dublin, ray.ekins@tudublin.ie
}

See next page for additional authors

Follow this and additional works at: https://arrow.tudublin.ie/archastrocon

Part of the Elementary Particles and Fields and String Theory Commons, and the Materials Science and Engineering Commons

\section{Recommended Citation}

Gorman, D., S. Jerrams, et al. (2011). "Creating a uniform magnetic field for the equi-biaxial physical testing of magnetorheological elastomers; electromagnet design, development and testing." ECCMR VII The 7th European Conference on Constitutive models for Rubber 2011.DIT, Dublin.

This Conference Paper is brought to you for free and open access by the Archaeoastronomy Research at ARROW@TU Dublin. It has been accepted for inclusion in Conference Papers by an authorized administrator of ARROW@TU Dublin. For more information, please contact arrow.admin@tudublin.ie, aisling.coyne@tudublin.ie,gerard.connolly@tudublin.ie.

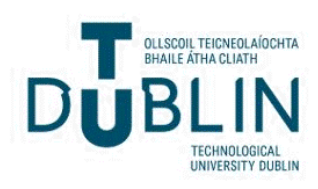




\section{Authors}

Dave Gorman, Stephen Jerrams, Ray Ekins, and Niall Murphy

This conference paper is available at ARROW@TU Dublin: https://arrow.tudublin.ie/archastrocon/3 


\title{
Creating a uniform magnetic field for the equi-biaxial physical testing of magnetorheological elastomers; electromagnet design, development and testing.
}

\author{
D Gorman, S Jerrams, R Ekins, and N Murphy
}

Centre of Elastomer Research, Dublin Institute of Technology, Republic of Ireland

\begin{abstract}
This paper investigates a method to provide the magnetic field requirements for physical testing of magnetorheological elastomers (MREs) subjected to equi-biaxial loading using the bubble inflation method.For accurate physical testing of MREs, detailed knowledge of the properties of the applied magnetic field is required. To obtain reliable data it is essential to determine the strength, uniformity and directionality of flux density. A Halbach cylinder array can produce a magnetic field of approximately uniform flux density in one direction for a reference plane perpendicular to the direction. However, it is limited by the fixed field strength. To overcome this significant limitation, an electromagnetic array based on the geometry of a Halbach cylinder is proposed. This electromagnetic array will be capable of generating a uniform magnetic field, for the reference plane and in the perpendicular direction, that is capable of having the flux density varied to offer a range of field strengths for tests on different elastomer samples.FEA simulations of uniform electromagnetic arrays have been modelled. Ultimately, a model is offered that simulates the behaviour of an electromagnetic array and the capability to generate a uniform magnetic field with different flux densities and directionality over the required volume. The advantages and disadvantages of an electromagnetic array over a fixed strength Halbach cylinder were investigated and a detailed comparison of both was carried out. Preliminary tests have been conducted on prototype electromagnets and the measured magnetic fields have been found to be in agreement with the FEA model. In addition to the magnetic field experiments, tests have been carried out on a compressed air cooling system to allow continuous operation of the electromagnets for the duration of a fatigue test without test samples becoming overheated and chemical degradation occurring. These tests are also necessary to establish conditions where there is minimal drop in field strength due to the increased resistance associated with temperature increases during prolonged dynamic testing. In conclusion a design for an electromagnetic array for the equi-biaxial testing of MREs is presented along with proposals for further testing to fully develop the array and establish standard dynamic test procedures for the material.
\end{abstract}

\section{INTRODUCTION}

MRE's are smart elastomers which change physical characteristics in the presence of a magnetic field (A. Boczkowska, 2009). These changes are due to ferromagnetic particles (usually iron) in the elastomer moving to align with the applied magnetic field (G.V. Stepanov, 2007). There are two types of MRE's, Isotropic and Anisotropic (Zsolt Varga, 2006). The difference between the two types is due to alternative curing processes. For Isotropic MRE's the ferromagnetic particles are added during curing and no external magnetic field is applied resulting in a random approximately uniform distribution. In the anisotropic case, an external magnetic field is applied during the curing process resulting in the fer- romagnetic particles moving in the gel to form aligned chains and being locked into this arrangement once the curing process is completed and the elastomer is formed.

The majority of experimental evaluation and testing of MRE's has been carried out on uniaxially loaded samples with the magnetic field assumed to be homogeneous in both flux density and directionality over the entire sample volume. Due to the larger sample volume required for the equi-biaxial physical testing of elastomers using bubble inflation (N Murphy, 2007), generating a magnetic field which maintains the necessary uniformity requires a more complex magnetic array.

A magnetic field of uniform strength is required as the MR (magnetorheological) effect is due to the 
magnetic iron particles in the MRE attempting to align in the direction of the applied magnetic field (Bica, 2009). This alignment is caused by the interactions of magnetic dipoles (G.V. Stepanov, 2007). The force on a magnetic dipole of moment $(\mathrm{m})$ in a magnetic field (B) is given in equation 1.1 (I.S. Grant, 1990).

$\operatorname{Fgrad}(\mathrm{m} . \mathrm{B})$

As the force which causes the alignment of particles is dependent on the magnetic field, the MR effect is dependent on the magnetic field strength. Therefore a non uniform strength field would result in an altered alignment and change in mechanical properties over the volume of the sample.

Uniform magnetic field direction is required for testing of MREs in both uniaxial and equi-biaxial cases, as the directional alignment of the field lines, applied force, and particle chains (Anisotropic MREs only) influence the material behaviour in a magnetic field. Both (Zsolt Varga, 2006) and (A. Boczkowska) found that a magnetic field applied parallel to the particle chains produces a larger MR effect compared with a field of the same flux density applied perpendicular to the particle chains.

A Halbach cylinder is a permanent magnet array arranged in a cylinder to produce a uniform field inside the cylinder and zero field outside (Coey, 2002). The Halbach cylinder produces a field of uniform strength and field lines in the required direction as shown in figure 1 but the fixed field strength limits its use as an effective device over a full range of test requirements.

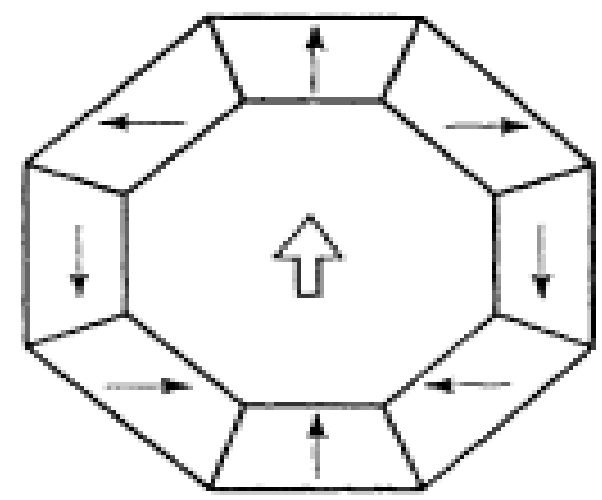

Figure 1 Halbach Cylinder (Coey, 2002)

Hills (B.P. Hills, 2005) developed an open access Halbach array to allow greater access to the sample while under NMR (nuclear magnetic resonance) imaging. This more open design came at the expense of both field strength and uniformity. A schematic of the Hills design is shown in figure 2.

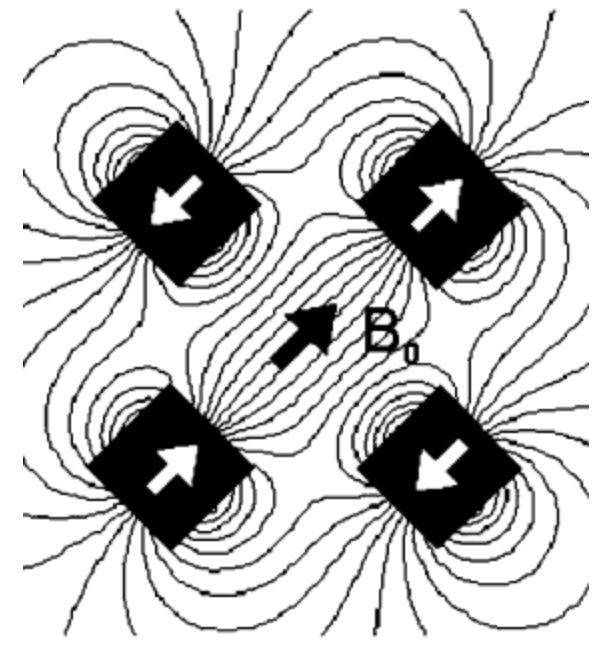

Figure 2 Open access Halbach array (B.P. Hills, 2005)

In order to provide a more comprehensive testing system which allows for a variable field strength, an equivalent arrangement of electromagnets is required. There are two significant problems with using electromagnets in preference to permanent magnets. These are, the electric power requirement and providing adequate cooling of the system (Montgomery, 1963). To generate a static magnetic field, a constant source DC voltage is required as field is proportional to current. It is also necessary to operate the electromagnetic array at a constant temperature as resistance is proportional to temperature and increases in temperature cause a drop in current and magnetic field for a given voltage.

\section{EXPERIMENTAL METHODS AND RESULTS}

\subsection{Modelling the magnetic field}

An FEA model was created using Finite Element Method Magnetics, FEMM4.2 (Meeker). This software was used to solve magnetic equations in which the fields are time-invariant. The field intensity $(\mathrm{H})$ and flux density (B) must obey equations 2.1 and 2.2

$\nabla \times \mathrm{H}=\mathrm{J}$

$\nabla \times \mathrm{B}=0$

Each material is modelled based on its magnetization curve which can be generated by plotting values determined from equation 2.3

$\mathrm{B}=\mu \mathrm{H}$

Equation 2.3 is non linear for magnetic materials as permeability $(\mu)$ is a function of B

The FEMM software has been use in the design of a magnetic system for the testing of MRFs (magnetorheological fluids) by (S.A. Mazlan, 2009). The 
results obtained by the simulations where in close agreement with the measured field.

The initial stage of the design method was to create an electromagnetic model based on the open access design presented by Hills (B.P. Hills, 2005) and shown in figure 2. An electromagnetic version of this arrangement was produced using 4 identical iron core electromagnets each with 1500 turns of $1 \mathrm{~mm}$ copper wire drawing a current of 15amps. The field produced by such an arrangement is presented in figure 3 and the area inside the coils is $80 \mathrm{~mm} \mathrm{x}$ $80 \mathrm{~mm}$. To obtain the same magnetic orientation as Hills' design, current in the two non central coils flows in the opposite direction to the other two coils.

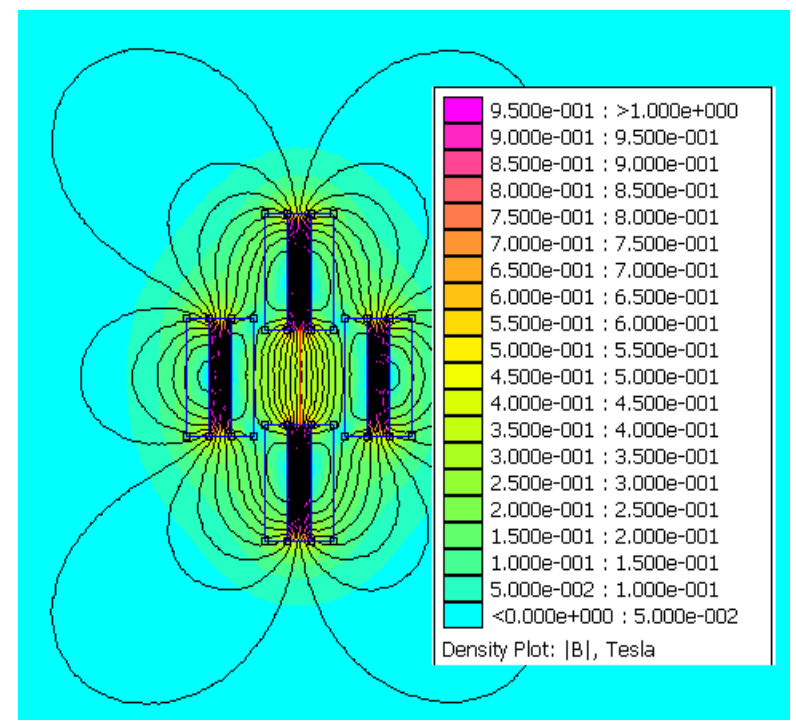

Figure 3 electromagnetic array based on Hills' design

The field generated along the central line in figure 3 is shown graphically in figure 4 .

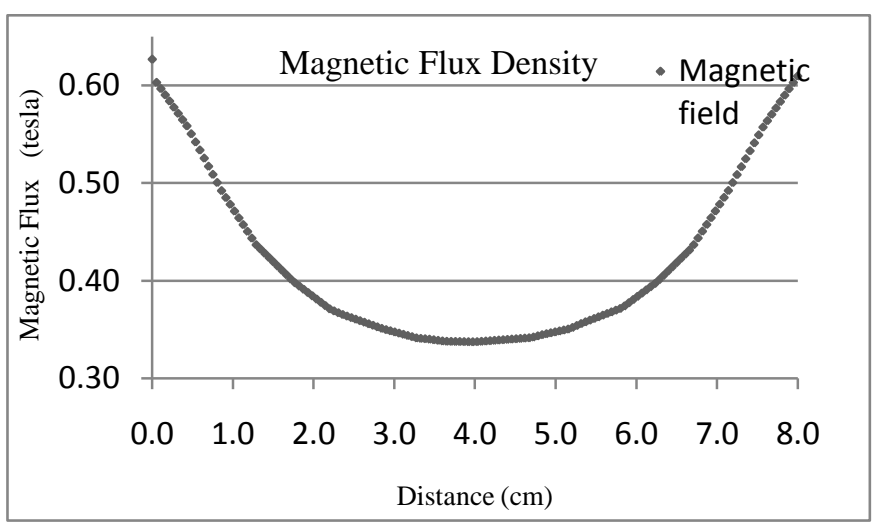

Figure 4 magnetic flux of array shown in figure 3

It is clear from the data shown in figures 3-4 that a simple replication of Hills opens access Halbach cylinder will not produce a field of uniform flux density to allow for the testing of MREs under equibiaxial conditions.
A series of modifications were made to the basic design shown in figure 3 and these are presented in figure 5 .

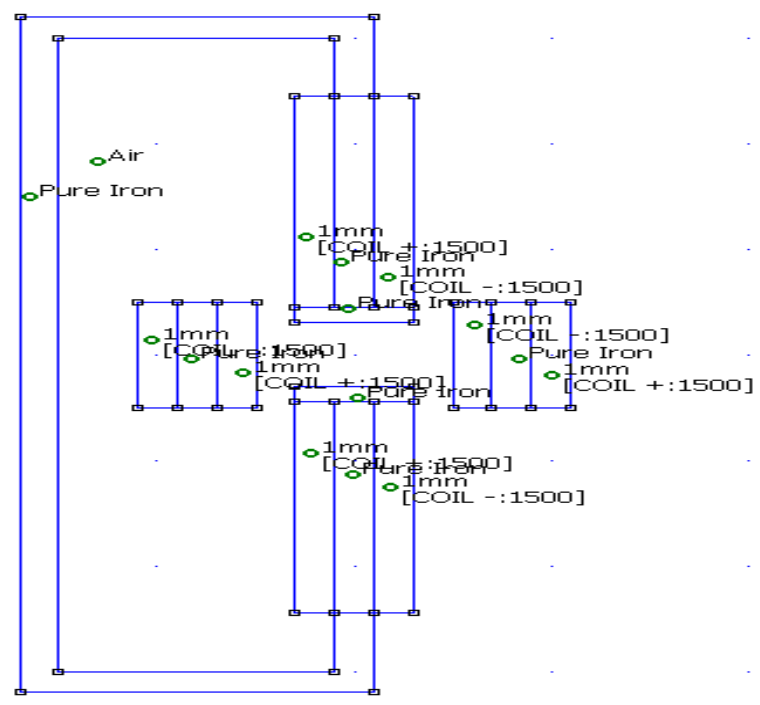

Figure 5 Proposed array

Figure 5 shows the changes made to the array. These include the connection of the two central electromagnets by an iron structure. This iron structure forms a pathway for the flux line to follow, thus increasing field strength.

The second notable change is the elongation of the central magnets. This allows for the requisite number of turns to be made with less wire, reducing the resistance of the coil and creating a more efficient electromagnet.

This modification was not applied to the other two electromagnets as their position was maintained to provide a field with uniform direction and they have less of an effect on the overall flux density.

The final changes where the addition of iron pole pieces to the central elongated electromagnets. This had the effect of increasing the uniformity of the field but also reduces the area between the coils to $80 \mathrm{~mm} \times 60 \mathrm{~mm}$.

The magnetic field produced by this modified array is shown in figure 6. All electromagnets had a current of 15amps with 1500turns. 


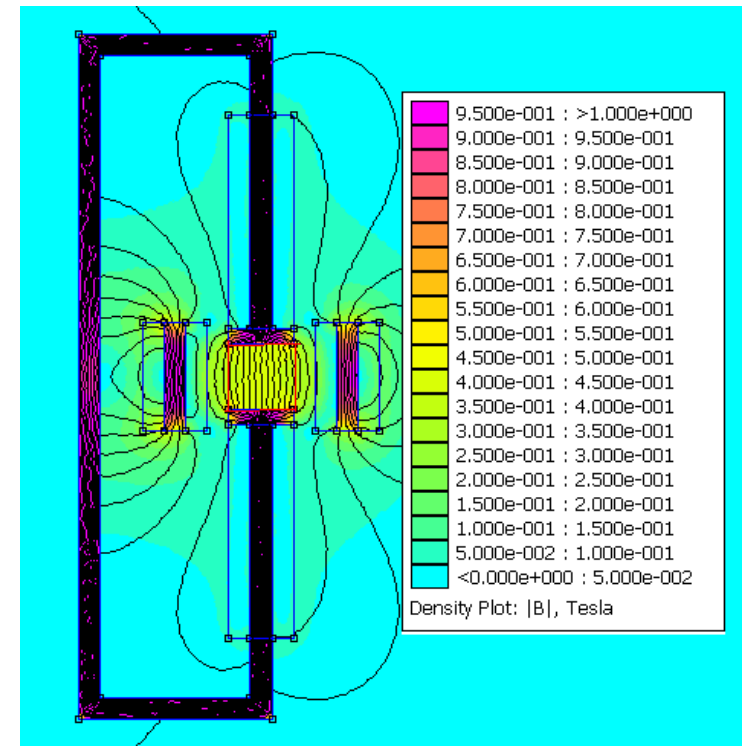

Figure 6 Simulated array field

The field flux density in the $60 \mathrm{~mm} \times 60 \mathrm{~mm}$ square highlighted in figure [6] is shown graphically in figure 7

\section{B field}

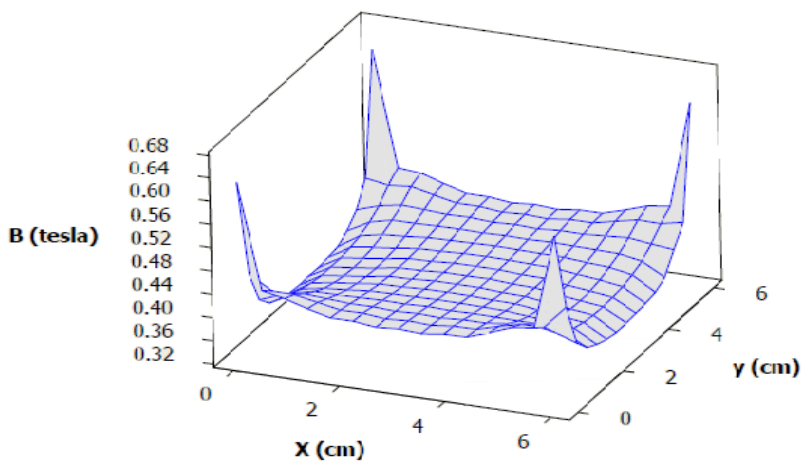

Figure 7 Magnetic Flux density of array shown in figure 6

It can be seen from figures 6-7 that the array shown in figure 5 is capable of producing a field with both the required uniformity of direction and flux density for the testing of MREs using bubble inflation.

\subsection{The simulated and generated magnetic fields}

To verify that the array presented in figure 5 is capable of producing the magnetic field simulated in figures 6-7, one of the central elongated electromagnets was tested and the actual measured magnetic field was compared with the simulated field produced by such a coil modelled with FEMM software.

The model of the prototype is shown in figure 8

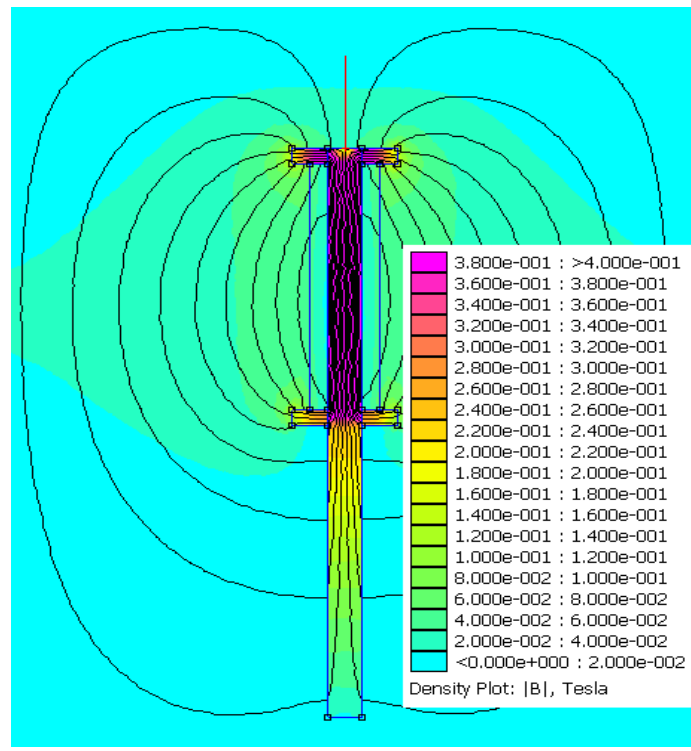

Figure 8 Simulated prototype field

The field produced along the line $\mathrm{v}$ distance from the coil and the actual field measured using a hall probe are shown in figure 9

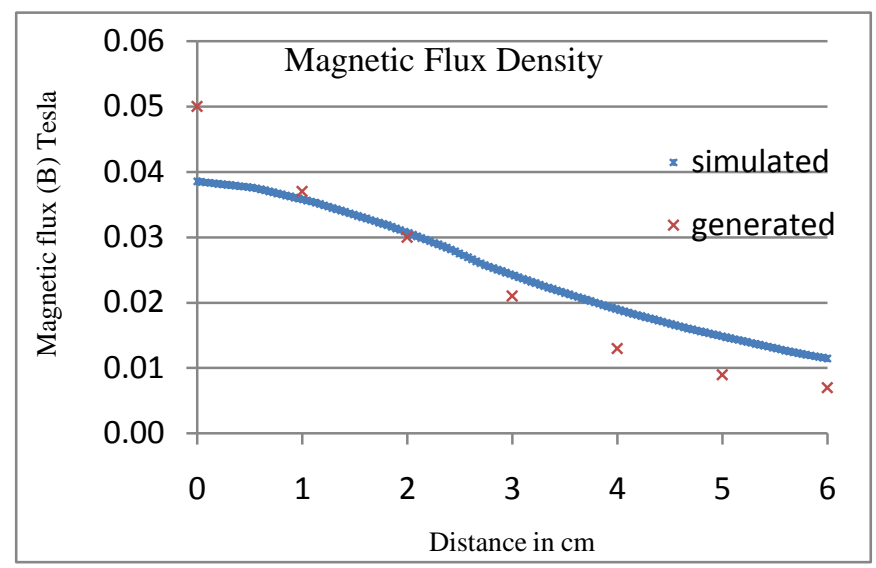

Figure 9 simulated $\mathrm{v}$ generated field from prototype

\subsection{Magnetic Array Cooling}

To ensure that the magnetic array operates with a constant current it must be held at a fixed temperature. This is achieved by cooling the electromagnet with compressed air via intakes and vents incorporated into the pole pieces of the magnets. (Outer radius $60 \mathrm{~mm}$ inner, radius $20 \mathrm{~mm}$ ). These are shown in figure 10

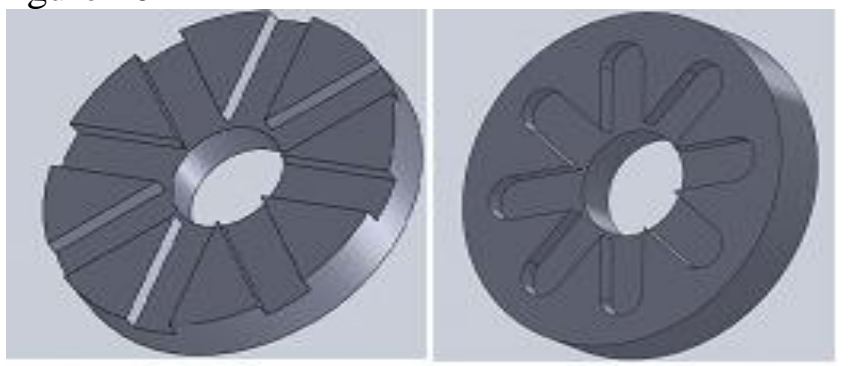

Figure 10 Pole Pieces 
The left image in figure 10 shows the vents from which the hot air exits the magnet and is the pole piece facing the sample. The right image in figure 10 shows the air intake system. A hole is drilled through one of the eight grooves in the same plane as the axis of the central bore. The air flow enters through this hole and then flows into the other seven grooves and from them down cooling channels incorporated into the windings and out through the vents.

Figure 11 shows the effectiveness of the cooling system.

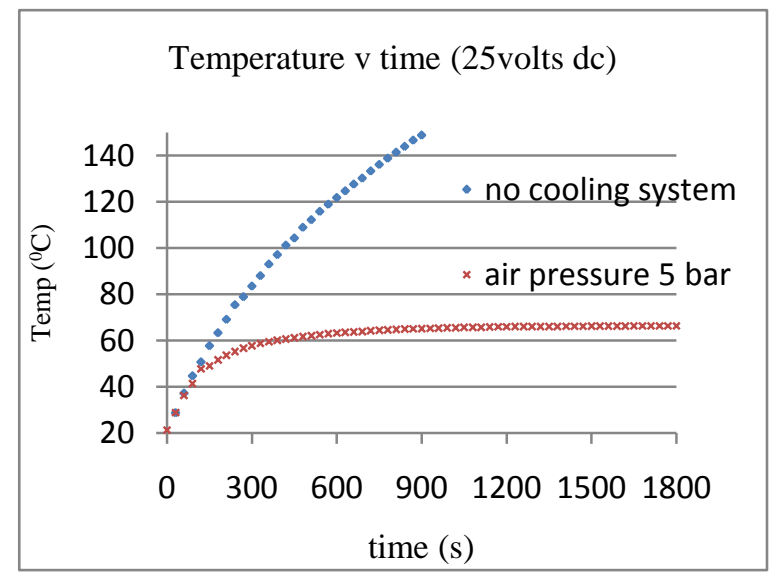

Figure 10 Temperature $v$ time

When the magnet was powered from a $25 \mathrm{~V}$ supply without cooling, a temperature of $140^{\circ} \mathrm{C}$ was reached after fifteen minutes continuous running. This was accompanied by a reduction in current from 6.84amps to 4.64amps. However, when the cooling system was actuated, a steady state was achieved at $66^{\circ} \mathrm{C}$ degrees with a current of 6.12 amps.

\section{CONCLUSIONS}

The electromagnetic array presented in figure 5 is capable of producing a suitable field for the equibiaxial physical testing of MREs. The field produced is uniform in both the required flux density and directionality of the field lines similar to that produced by a Halbach cylinder. The advantage of such a design over a Halbach cylinder is that electromagnetic arrays can produce a uniform field over a range of flux densities. For this design, the flux density can be varied in the range $0-420 \mathrm{mT}$.

By comparison with a permanent magnet design the disadvantages of any electromagnetic design, are the requirements for constant input of energy and the removal of heat energy.

The heating problems can be overcome by the proposed cooling system and a steady state can be maintained for the duration of a fatigue test on a magnetorheological elastomer sample.

\section{PROPOSED FURTHER WORK}

The prototype will be evaluated at higher currents and once the design is proven, the full electromagnetic array will be manufactured. This will lead to an extensive test programme investigating the dynamic properties of a range of MREs subjected to complex loading. In particular, fatigue resilience, stress softening and set will be determined for MREs based on natural rubber (NR), silicone and ethylene propylene diene monomer (EPDM) matrices with a range of ferromagnetic particle sizes and volume fractions.

\section{REFERENCES}

Bica I. Compressibility modulus and principal deformations in magneto-rheological elastomer: The effect of the magnetic field: J. Ind. Eng. Chem., 2009.

Boczkowska A., Awietjan S. Mechanical properties of magnetorheological elastomers

Boczkowska A., Awietjan S. Smart composites of urethane elastomers with carbonyl iron - [s.1.] : Journal of material science, 2009. - 15 : Vol. 44.

Coey J.M.D. Permanent magnet applications - [s.1.]:

Elsevier, 2002. - 248 (441-456) :Journal of magnetism and Magnetic Materials.

Grant I.S., Phillips W.R. Electromagnetism

[Book]. - [s.1.] : Wiley, 1990. - Vol. 2nd edition. chapter 4 p131

Hills B.P., Wright K.M., Gillies D.G. A low field, low cost Halbach magnet array for open access NMR [s.1.] : Journal of Magnetic Resinance, 2005. - 175 (336-339).

Mazlan S.A. Issa A., Chowdhury H.A, Olabi A.G. Magnetic circuit design for the squeeze mode experiments on magnetorheological fluids. : Materials and Design, 2009. - 30 1985-1993.

Meeker D. [SoftwareOnline]. - http://www.femm.info.

Montgomery D.B. The Generation of High Magnetic Fields Reports on progress in physics- 1963. Vol. 26

Murphy N., Hanley J., Ali H., Jerrams S., The effect of specimen geometry on the multiaxial deformation of elastomers: Taylor \& Francis, 2007. - ECCMR V :Paris

Stepanov G.V., Abramchuk S.S., Grishin D.A., Nikitin L.V., Kramarenko E Yu., Khokhlov A.R. Effect of a homogeneous magnetic field on the viscoelastic behaviour of magnetic elastomers. - [s.1.] : Elsevier, 2007. - 488-495 : Vol. Polymer 48.

Varga Z., Filipcsei G., Zrı'nyi M. Magnetic field sensitive functional elastomers with tuneable elastic modulus : Elsevier, 2006. - 227-233 : Vol. Polymer 47. 